\author{
Revista Brasileira de Tecnologia \\ Agroindustrial
}

\title{
UTILIZAÇÃO DE FARINHA MISTA DE TRIGO E QUINOA NA ELABORAÇÃO DE BOLOS
}

\section{USE OF WHEAT AND QUINOA COMPOSITE FLOUR IN CAKE MAKING}

\author{
João Tomaz da Silva Borges ${ }^{1}$; Mônica Ribeiro Pirozi ${ }^{2}$; Juliana Gonçalves Vidigal ${ }^{3}$ \\ Cláudia Denise de Paula ${ }^{4}$; Natanielli Alves de Sousa e Silva ${ }^{5}$ \\ ${ }^{1}$ Instituto Federal de Minas Gerais - IFMG - São João Evangelista-MG - Brasil joao.tomaz@ifmg.edu.br \\ ${ }^{2,5}$ Universidade Federal de Viçosa - UFV - Viçosa, MG - Brasil mpirozi@ ufv.br \\ ${ }^{3}$ Instituto Federal Fluminense - IFF - Bom Jesus do Itabapoana - RJ - Brasil \\ ${ }^{4}$ Universidade de Córdoba, Montería - Colômbia.
}

\begin{abstract}
Resumo
O objetivo do presente trabalho foi avaliar a viabilidade tecnológica e sensorial do uso de farinhas mistas de trigo e quinoa nas proporções de 100:00 (F0), 90:10(F10) e 80:20 (F20) na elaboração de bolos. A farinha de quinoa (FQ) apresentou granulometria mais grosseira quando comparada à farinha de trigo (FT), entretanto, não foram observadas variações expressivas nas misturas estudadas. Foi verificado, pela análise farinográfica, que a absorção de água e o tempo de máximo desenvolvimento aumentaram $(p<0,05)$ com o incremento no teor de $F Q$. Não houve variações $(p<0$ '05) na densidade específica da massa crua, volume, simetria e contorno dos bolos F10 e F20 em comparação com FO. Os componentes $L^{*} e b^{*}$ não variam $(p>0,05)$ entre as formulações, todavia, os valores de $a^{*}$ demonstraram tendência $(p<0,05)$ ao escurecimento com o aumento de quinoa na mistura. A análise sensorial não apresentou diferença $(p>0,05)$ entre as formulações com $10 \%$ e $20 \%$ de FQ, demonstrando boa aceitação dos bolos pelos consumidores. O emprego de até $20 \%$ de quinoa não modificou a aceitação dos atributos aroma, cor, aparência, sabor e textura do bolo, sendo viável a utilização dessa porcentagem para substituição parcial da farinha de trigo na elaboração de bolos.
\end{abstract}

Palavras-chave: trigo; quinoa; bolo; avaliação física; aceitação sensorial.

\section{Introdução}

Grande parte dos produtos de panificação é composta por ingredientes que desempenham funções específicas no processo de formação da massa. Embora os constituintes possam variar em grau de importância no processo de fabricação, todos exercem determinada função. Muitas vezes, a 
maior ou menor importância desses componentes está associada com a quantidade adicionada à massa e ao tipo de produto final desejado (PYLER e GORTON, 2009).

Na década de 60, a utilização de farinhas mistas provenientes de duas ou mais matériasprimas tinha como objetivo a substituição parcial da farinha de trigo para redução das importações desse cereal. Posteriormente, as pesquisas com essas misturas foram direcionadas para a melhoria da qualidade nutricional de produtos alimentícios e para suprir a necessidade dos consumidores por produtos diversificados.

Vários fatores devem ser considerados na utilização de farinhas mistas para produção de alimentos. As características de farinhas sucedâneas devem reduzir, ao máximo, os efeitos deletérios da substituição sobre as características tecnológicas, a fim de se obter alimentos com características nutricionais, sensoriais e economicamente viáveis, que permitam sua real utilização pelos consumidores (BORGES et al., 2011; ALMEIDA et al., 2011).

Entre os produtos de panificação, o bolo vem adquirindo crescente importância no que se refere ao consumo e comercialização no Brasil. O desenvolvimento tecnológico possibilitou mudanças nas indústrias, transformando a produção de pequena para grande escala (GORGÔNIO et al., 2011). Embora não constitua alimento básico como o pão, o bolo é aceito e consumido por pessoas de qualquer idade. Trata-se de produto obtido pela mistura, homogeneização e cozimento conveniente de massa preparada com farinhas, fermentadas ou não e outras substâncias alimentícias (como, por exemplo, leite, ovos e gordura). A farinha de trigo constitui o principal componente das formulações por fornecer a matriz em torno da qual os demais ingredientes são misturados para formar a massa (CAUVAIN e YOUNG, 2009; GUTKOSKY et al., 2011).

Vários estudos têm sido realizados com a finalidade de melhorar o valor nutritivo de bolos, principalmente quanto ao conteúdo de minerais, vitaminas e fibras alimentares. Bolos obtidos a partir de farinhas mistas, farinhas integrais ou com adição de micro ou macronutrientes têm despertado a atenção de consumidores por sua contribuição no suprimento de necessidades nutricionais diárias ou por disponibilizar substâncias com alegações de propriedades funcionais que previnem ou auxiliam o tratamento de doenças, como fibras, ácidos graxos essenciais, minerais, substâncias prebióticas, dentre outros (MORAES et al., 2010; GUIMARÃES et al., 2010; COELHO e WOSIACK, 2010; GÓMEZ et al., 2010; SCHMIELE et al., 2011; HERA et al., 2012).

A quinoa (Chenopodium quinoa Willd.) é uma planta alimentícia da região dos Andes, classificada como dicotiledônea, sendo denominada por alguns autores como pseudocereal. Esta planta tem elevado valor econômico por ser considerada componente potencial na ração animal e na alimentação humana. Devido à sua alta qualidade nutricional, tem despertado a atenção de pesquisadores em várias partes do mundo, sendo desta forma utilizada em blends (denominado 
também de farinhas mistas ou compostas, obtida a partir de duas ou mais matérias-primas) com farinha de trigo para produtos de panificados, e também utilizada na elaboração de isentos de glúten para portadores de doença celíaca (DANELLI et al., 2010; CALDERELLI et al., 2010; REPOCARRASCO-VALENCIA e SERNA, 2011).

O presente trabalho tem por objetivo avaliar a viabilidade tecnológica e sensorial do uso da farinha trigo em mistura com farinha de quinoa na elaboração de bolos.

\section{Material e Métodos}

O presente trabalho foi realizado nos Laboratórios de Processamento e Análise Sensorial de Alimentos do Instituto Federal do Espírito Santo, campus Venda Nova do Imigrante e de Panificação do Departamento de Tecnologia de Alimentos, Universidade Federal de Viçosa, MG.

\section{Matérias-primas e farinhas mistas}

Foram utilizadas como matérias-primas farinha de trigo especial (FT) e farinha integral de quinoa (FQ), adquiridas no comércio de Venda Nova do Imigrante, Espírito Santo. As farinhas mistas foram preparadas em misturadora vertical, marca Brasil, modelo 56 RC 6504, com batedor tipo gancho, pela substituição parcial de FT por FQ nas proporções de 100:00 (F0, Controle), 90:10 (F10) e 80:20 (F20). As matérias-primas foram colocadas no tacho coletor do equipamento e mantidas sob agitação constante, com o registro de velocidade posicionado no número 2, segundo manual do fabricante, por 10 minutos, para garantir melhor uniformidade da mistura.

\section{Classificação granulométrica}

A distribuição granulométrica da FQ, F0, F10 e F20 foi determinada por meio de equipamento Ro-Tap e conjunto de seis peneiras arredondadas, com aberturas da malha de 590, 420, 350, 297, 250 e 210 micrômetros e o fundo (AOAC, 1995). Em seguida, as quantidades retidas em cada peneira foram pesadas e expressas em percentagens.

\section{Farinografia}

Utilizou-se farinógrafo Brabender para a farinografia da farinha de trigo (F0) e misturas F10 e F20, obtendo-se, inicialmente, a curva de titulação e, posteriormente, a curva padrão (AACC, 1983). 


\section{Preparo dos bolos}

Para produção dos bolos, além das farinhas F0, F10 e F20, foram utilizados açúcar refinado, ovos, margarina cremosa com sal, fermento químico e leite integral, adquiridos no comércio de Venda Nova do Imigrante, Espírito Santo.

Os bolos Controle (F0), F10 e F20, provenientes das farinhas F0, F10 e F20 respectivamente, foram preparados pela mistura do ovo, do açúcar e da margarina por 1 minuto em batedeira até formação de uma massa homogênea (Tabela 1). Adicionou-se a farinha e o leite, batendo por 2 minutos. Ao final, acrescentou-se o fermento químico e a massa foi misturada por mais 1 minuto, sendo, posteriormente, colocada em forma forrada com papel manteiga e assada em forno pré-aquecido a $180^{\circ} \mathrm{C}$. O tempo de assamento foi de, aproximadamente, 35 minutos.

Tabela 1 - Ingredientes utilizados nas diferentes formulações dos bolos F0, F10 e F20

\begin{tabular}{lccc}
\hline \multirow{2}{*}{ Ingredientes* } & \multicolumn{3}{c}{ Bolos } \\
\cline { 2 - 4 } & F0 & F 10 & F 20 \\
\hline Farinha de Trigo & 100 & 90 & 80 \\
Farinha de quinoa & - & 10 & 20 \\
\hline Açúcar & 75 & 75 & 75 \\
Ovo & 40 & 40 & 40 \\
Margarina & 30 & 30 & 30 \\
Fermento & 4 & 4 & 4 \\
Leite & qsp** & qsp & qsp \\
\hline *Porcentagem dos ingredientes em relação a 100\% do peso total de farinha mista. \\
**Quantidade suficiente para
\end{tabular}

\section{Densidade específica da massa crua}

A densidade específica da massa crua foi determinada, relacionando o peso da massa crua de cada formulação e o seu volume, sendo o resultado expresso em g/mL (ZAMBRANO et al., 2005).

\section{Análises físicas do bolo}

Os bolos F0, F10 e F20 foram submetidos à avaliação de volume, contorno e simetria de acordo com o método 10-90 da AACC (2000).

A quantificação objetiva de cor foi feita por meio de um colorímetro triestímulo, com leitura direta de reflectância das coordenadas de cromaticidade "L" (luminosidade), "a" (tonalidades de vermelho a verde) e "b" (tonalidades de amarelo a azul), empregando-se a escala Hunter-Lab. Neste sistema de cor, corrigido pela CIELab, os valores L* (luminosidade) variam de zero (preto) a 100 (branco), os valores de $\mathrm{a}^{*}$ variam de $-\mathrm{a}^{*}$ (verde) até $+\mathrm{a}^{*}$ (vermelho), e os valores de $\mathrm{b}^{*}$ variam de $\mathrm{b}^{*}$ (azul) até $+\mathrm{b}^{*}$ (amarelo) (HUNTERLAB, 1998). Os valores correspondentes às coordenadas de 
cromaticidade $\mathrm{L}^{*}, \mathrm{a}^{*} \mathrm{e} \mathrm{b}^{*}$ foram obtidos por meio de leitura direta das fatias de bolo submetidas ao colorímetro. As diferenças de cor $\left(\Delta \mathrm{E}^{*}\right)$ entre as formulações de bolo contendo as farinhas mistas (F10 e F20) e a Controle (F0) foram calculadas de acordo com a equação:

$$
\Delta \mathrm{E}^{*}=\left[\left(\Delta \mathrm{L}^{*}\right)^{2}+\left(\Delta \mathrm{a}^{*}\right)^{2}+\left(\Delta \mathrm{b}^{*}\right)^{2}\right]^{1 / 2} \quad\left\{\begin{array}{l}
\text { Onde: } \\
\Delta \mathrm{E}^{*}= \\
\text { diferença entre cada parâmetro de cor das } \\
\text { farinhas mistas com a farinha de trigo (padrão). }
\end{array}\right.
$$

\section{Análise sensorial}

Os bolos F10 e F20 foram avaliados quanto à aceitação sensorial considerando os atributos aroma, cor, aparência, sabor e textura, utilizando-se de escala hedônica estruturada de nove pontos (MINIM, 2010).

As amostras de ambos os bolos foram servidas, monadicamente, a 117 julgadores não treinados (consumidores), com idade entre 7 e 71 anos, de ambos os sexos, residentes na cidade de Venda Nova do Imigrante-ES, sendo selecionados para realizar a análise com base no hábito de consumir bolo.

\section{Delineamento experimental e análise estatística}

Empregou-se delineamento inteiramente casualizado com 3 formulações (F0, F10 e F20) e 3 repetições, totalizando 9 unidades experimentais. Todas as análises foram feitas em triplicata. Para comparar as médias das análises farinográficas, densidade específica da massa crua e de cor dos bolos em função do nível de substituição de FT pela FQ usou-se a análise de variância (ANOVA) e o teste de Dunnett $(\mathrm{p}<0,05)$. Os resultados do teste de aceitação sensorial foram submetidos à ANOVA, sendo as médias dos tratamentos comparadas pelo teste $\mathrm{F}(\mathrm{p}<0,05)$. Todas as análises foram realizadas utilizando-se o programa Statistical Analysis System (SAS, 1996) versão 9.1, licenciado para a Universidade Federal de Viçosa, Minas Gerais, 2009.

\section{Resultados e Discussão}

Distribuição do tamanho de partículas das farinhas de quinoa, de trigo e mistas

A característica granulométrica da matéria-prima constitui aspecto relevante na elaboração de massas alimentícias e produtos de padaria, pois a distribuição adequada de partículas permite maior uniformidade do produto elaborado. A Figura 1 apresenta a análise granulométrica das farinhas FT (F0), FQ, F10 e F20. 
Figura 1 - Distribuição (\%) do tamanho de partículas da farinha de trigo (FT), quinoa (FQ) e farinhas mistas F10 e F20

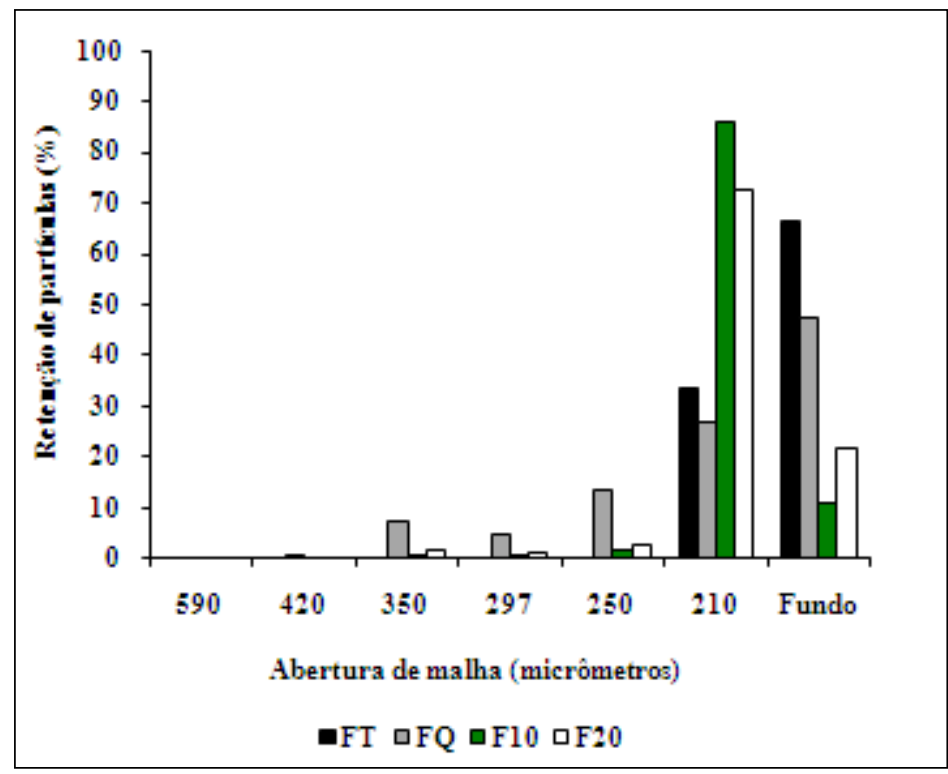

A FQ apresentou granulometria mais grosseira quando comparada à FT. Não foram observadas variações expressivas nas misturas, considerando que maior parte passou pela peneira de 250 micrômetros, indicando características semelhantes à FT, conforme estabelecido pela Portaria n. 354, de 18 de julho de 1996 (ANVISA, 2012).

A granulometria influencia diretamente a capacidade de absorção de água, as características sensoriais (como aparência, sabor e textura) e o tempo de cozimento das massas alimentícias. Entretanto, sua influência nas propriedades de bolos ainda não foi totalmente elucidada, embora seja aceito que partículas finas e uniformes promovem maior dispersibilidade da farinha na massa. (BORGES et al., 2006; SILVA et al., 2009; PYLER e GORTON, 2009).

O tamanho de partícula de farinhas, após a moagem, constitui aspecto importante no preparo de massas alimentícias e demais derivados, tendo em vista que, uma maior uniformidade da granulometria permite a elaboração de um produto final de melhor qualidade sensorial, principalmente textura, sabor e aspecto visual, pois o alimento absorve água de forma homogênea, resultando no cozimento uniforme da massa (SILVA et al., 2009).

\section{Análise farinográfica}

A Tabela 2 apresenta os valores obtidos na análise farinográfica das amostras de farinhas mistas. A farinografia avalia a qualidade de uma farinha no que se refere a sua capacidade de absorver água, desenvolvimento de glúten e resistência, durante a mistura/amassamento (COSTA et 
al., 2008). Em bolos, a mais alta absorção de água é o elemento farinográfico mais importante, e se considera o menor desenvolvimento de glúten como ideal, neste caso, uma vez que está associado ao produto final com melhor textura no miolo (PYLER e GORTON, 2009). De uma forma geral, as amostras F10 e F20 mostraram melhor qualidade do que F0, quando considerados os valores de absorção de água (AA) mais elevados e menores tempo de chegada (TC), estabilidade (ES), tempo de desenvolvimento da massa (TDM) e índice de tolerância à mistura (ITM).

Tabela 2 - Absorção de água e propriedades de mistura da massa de farinha de trigo e farinhas mistas

\begin{tabular}{llllll}
\hline \multicolumn{1}{c}{ FM } & \multicolumn{1}{c}{ AA $(\%)$} & \multicolumn{1}{c}{ TC $($ min) } & ES (min) & \multicolumn{1}{c}{ TDM (min) } & \multicolumn{1}{c}{ ITM (UF) } \\
\hline F0 & $60,6 \pm 0,00$ & $1,1 \pm 0,09$ & $15,2 \pm 0,47$ & $16,4 \pm 1,73$ & $38,9 \pm 1,92$ \\
F10 & $63,7^{*} \pm 0,00$ & $2,1^{*} \pm 0,19$ & $10,8^{*} \pm 0,88$ & $10,6^{*} \pm 0,26$ & $50,0^{*} \pm 3,18$ \\
F20 & $64,3^{*} \pm 0,00$ & $2,1^{*} \pm 0,07$ & $10,9 * \pm 0,09$ & $11,1^{*} \pm 0,10$ & $60,0^{*} \pm 0,58$ \\
\hline
\end{tabular}

*Representa diferença significativa em relação ao Controle pelo teste de Dunnett $(\mathrm{p}<0,05) . \mathrm{AA}=$ absorção de água; TC $=$ tempo de chegada; ES = estabilidade; TDM = tempo de desenvolvimento da massa; ITM = índice de tolerância à mistura

De acordo com os resultados obtidos, nota-se que houve um incremento nos níveis de AA quando parte da farinha de trigo foi substituída pela quinoa. Este valor indica a qualidade da farinha de trigo refletida pela capacidade de intumescimento do glúten e o teor de amido danificado, que irá determinar a consistência da massa, pois a água reforça a união de gliadina e glutenina, dando origem ao glúten (GUTKOSKI et al., 2008). Neste caso, porém, o aumento de AA deve estar relacionado a elevados teores de fibra na farinha de quinoa (LOPES et al., 2010) quando comparada à farinha de trigo (NEPA, 2011), além de altos níveis de amido danificado na mistura, decorrentes das condições de moagem (GUTKOSKI et al., 2008). Hadnađev et al. (2011) também observaram um aumento significativo na AA de farinhas integrais quando comparada a farinha de trigo branca.

O TC aumentou com a adição de farinha de quinoa na mistura. Esse comportamento sugere influência da fibra presente na farinha de quinoa, na formação da rede de glúten (GEWEHR, 2010; GÓMES et al., 2010), além da própria diminuição relativa do teor total de glúten presente na massa de farinha mista.

Observou-se diminuição nos valores da ES da massa, indicando que a presença de quinoa reduziu a força da massa. Isto sugere menor tolerância ao amassamento ou à ação mecânica. Estes resultados corroboram com aqueles obtidos por Fernandes et al. (2008) ao substituírem 9\% e 12\% de farinha de trigo por farinha de casca de batata. Para os autores, com a diminuição da farinha de trigo e, consequentemente, do glúten, a farinha se torna mais fraca e menos tolerante à mistura. Existe uma correlação positiva entre a qualidade da proteína na farinha e a ES da massa; uma ES maior indica uma melhor qualidade das proteínas e, portanto, uma melhor qualidade da farinha. Para Indrani e Rao (2007) e Costa et al. (2008), a ES de uma massa é reconhecida como um 
parâmetro indicador de maior resistência ao amassamento e melhor qualidade tecnológica. Os valores de ES de massas dependem, em grande parte, do número de ligações cruzadas entre as moléculas de proteínas presentes no glúten, bem como da força destas ligações. As pontes de hidrogênio e as ligações de enxofre desempenham grande importância na formação da estrutura do glúten, pois formam uma rede tridimensional relacionada à extensibilidade, impermeabilidade ao gás, elasticidade e ao alto poder de absorção de água da massa.

O TDM indica a qualidade da proteína e, farinhas fortes normalmente requerem maiores valores deste quando comparadas às farinhas fracas (PYLER e GORTON, 2009; CAUVAIN e YOUNG, 2009). As duas concentrações de misturas de farinhas apresentaram menor TDM em comparação com a farinha de trigo pura, o que é característico de farinhas fracas, favoráveis à produção de bolos. A adição de farinha de quinoa torna o glúten menos elástico, alterando as propriedades reológicas da mistura já que suas proteínas não formam glúten.

Maiores valores de ITM foram obtidos com o aumento da concentração de farinha de quinoa, indicando o enfraquecimento da mistura para a formação de glúten. Esse comportamento ocorre, provavelmente, pelo aumento de fibra na massa, que age interrompendo fisicamente a estrutura contínua do glúten, bem como pelo seu enfraquecimento proporcionado com a adição de farinha de quinoa. Comumente, farinhas que apresentam boa tolerância à mistura revelam menor ITM (maior índice de tolerância indica farinha mais fraca).

Ao utilizar farinhas mistas ocorrem alterações nas propriedades físicas e tecnológicas, que podem ser favoráveis ou não, conforme o produto. No caso do bolo, a farinha fraca proporcionada pela mistura é desejável. A estrutura desse produto é mais leve, porosa, e requer menor desenvolvimento do glúten do que produtos como pão. No Brasil, a farinha empregada na produção de bolos é a mesma usada para fazer pães. Conforme Pyler e Gorton (2009), farinhas fortes podem ser enfraquecidas pela adição de outras farinhas, como a farinha de quinoa utilizada no presente estudo.

\section{Densidade específica da massa crua e análise física dos bolos}

As formulações contendo $10 \%$ e $20 \%$ de farinha de quinoa não diferiram (p>0,05) de F0 em relação à densidade específica da massa crua, volume, simetria e contorno dos bolos (Tabela 3). Conforme afirmam Pyler e Gorton (2009), a densidade constitui medida de incorporação e retenção de ar na massa de bolo e quanto menor seu valor, maior será a quantidade de ar incorporado à mistura. Valores de densidade altos desfavorecem o volume do produto e provocam a formação de miolo fechado, massudo e de mau aspecto. 
Tabela 3 - Densidade específica da massa crua; volume, simetria e contorno dos bolos

\begin{tabular}{ccccc}
\hline & \multirow{2}{*}{$\begin{array}{c}\text { Densidade } \\
\text { Bolos }\end{array}$} & \multicolumn{3}{c}{ Análises físicas } \\
\cline { 3 - 4 } & massa crua & Volume $(\mathbf{m m})$ & Simetria $(\mathbf{m m})$ & Contorno $(\mathbf{m m})$ \\
\hline F0 (Controle) & 0,9 & 155,4 & 0 & 12,3 \\
F10 & $0,9^{\mathrm{ns}}$ & $153,2^{\mathrm{ns}}$ & $0^{\mathrm{ns}}$ & $13,2^{\mathrm{ns}}$ \\
F20 & $0,9^{\mathrm{ns}}$ & $153,9^{\mathrm{ns}}$ & $0^{\mathrm{ns}}$ & $13,4^{\mathrm{ns}}$ \\
\hline *Representa diferença significativa em relação ao Controle pelo teste de Dunnett $(\mathrm{p}<0,05) .{ }^{\mathrm{ns}}$ Não significativo
\end{tabular}

*Representa diferença significativa em relação ao Controle pelo teste de Dunnett $(\mathrm{p}<0,05) .{ }^{\text {ns }}$ Não significativo

Os valores de volume encontrados demonstram que a incorporação de quinoa na formulação não prejudicou a estrutura proteica na massa dos bolos. Entretanto, percebe-se que concentrações mais elevadas de farinha de quinoa na mistura podem comprometer a estrutura do miolo, sendo necessário o uso de modificações no processo, ou mesmo o uso de aditivos, como por exemplo, emulsificantes, que minimizem seus efeitos na qualidade do produto final. $\mathrm{O}$ volume do bolo resulta do uso de clara de ovo e suas proteínas, importantes na produção de produtos com baixa densidade e elevada expansibilidade em virtude da capacidade de incorporar ar e formar espumas (YANG e FOEGEDING, 2009). Embora não significativos ( $p>0,05)$, os resultados obtidos de volume em F10 e F20 sugerem que a composição química da farinha de quinoa interferiu na formação e agregação da estrutura proteica ao redor das bolhas de ar na massa, contribuindo para a redução de volume do produto final. Para Kocer et al. (2007), Cauvain e Young (2009) e Schmiele et al. (2011), além da quantidade de ar incorporado na massa, o tamanho das bolhas de ar também é muito importante. Esse tamanho vai depender do raio, da tensão superficial e da pressão interna. Sabe-se que, quanto menor o raio maior é a pressão e que, geralmente, o tamanho da bolha de ar no produto final é 3 a 5 vezes maior em relação à massa. Foegeding et al. (2006) constataram que com a elevação da temperatura no forneamento, a desnaturação proteica e a gelatinização do amido determinam o volume do bolo, firmeza ou colapso de sua estrutura.

Os resultados da avaliação de simetria não variaram $(\mathrm{p}>0,05)$ de $0 \mathrm{~mm}$ em nenhum dos bolos analisados. O desenvolvimento simétrico e a qualidade de contorno de massa durante o forneamento constituem características importantes no processamento de produtos de panificação. Para bolos, a simetria ideal assume valor zero e o contorno deve ser positivo, significando crescimento uniforme da massa e manutenção estrutural durante a cocção, além de equilíbrio adequado de ingredientes na formulação e condições de processamento.

A forma ou simetria de produtos de panificação deve ser uniforme e bem definida, caso contrário indica manuseio e processamento inadequados. Simetria diferente de zero resulta, ainda, de massa muito dura com baixo teor de umidade, bem como fermentação inadequada, insuficiente ou manuseio incorreto e grosseiro (PYLER e GORTON, 2009). 
Os resultados referentes à colorimetria dos bolos encontram-se na Tabela 4. A análise objetiva dos componentes $\mathrm{L}^{*}, \mathrm{a}^{*}$ e $\mathrm{b}^{*}$ não mostrou variações entre as formulações, com exceção do componente $\mathrm{a}^{*}(\mathrm{p}<0,05)$.

Tabela 4 - Avaliação das coordenadas $\mathrm{L}^{*} \mathrm{a}^{*} \mathrm{~b}^{*}$ e diferença de cor (Delta $\mathrm{E}^{*}$ ) dos bolos F10 e F20 em comparação com F0

\begin{tabular}{llllc}
\hline \multicolumn{1}{c}{ Bolos } & $\mathbf{L}^{*}$ & $\mathbf{a}^{*}$ & $\mathbf{b}^{*}$ & Delta E \\
\hline F0 (Controle) & 73,2 & 3,1 & 23,2 & - \\
F10 & $73,9^{\text {ns }}$ & $2,4^{*}$ & $22,7^{\text {ns }}$ & 1,1 \\
F20 & $71,7^{\text {ns }}$ & $2,7^{*}$ & $23,1^{\text {ns }}$ & 1,6 \\
\hline
\end{tabular}

*Representa diferença significativa em relação ao Controle pelo teste de Dunnett $(\mathrm{p}<0,05) .{ }^{\text {ns }}$ Não significativo

Os valores de $\mathrm{L}^{*}$, que indicam a luminosidade da amostra na faixa de 100 (branco) a 0 (negro), não diferiram ( $\mathrm{p}>0,05)$ entre as amostras de bolo. Porém, pode-se observar que o aumento na adição de farinha de quinoa (20\%) favorece à diminuição da luminosidade do produto, sendo o oposto observado quando menores concentrações são incorporadas à mistura.

A coordenada $\mathrm{a}^{*}$, que indica a variação de verde $\left(-\mathrm{a}^{*}\right)$ a vermelho $\left(+\mathrm{a}^{*}\right)$, é um parâmetro importante para o estudo de escurecimento, pois a cor marrom resultante da degradação dos açúcares (reação de caramelização) ou reações enzimáticas (fenolases) representa uma combinação do verde e vermelho. Um maior escurecimento é representado por um tom mais avermelhado, ou seja, maior valor de $a^{*}$. Resultados semelhantes foram também obtidos por Gewehr (2010) e Alves et al. (2010) ao utilizarem quinoa na elaboração de pão de forma e pão de queijo, respectivamente.

A coordenada $b^{*}$, relacionada ao eixo que varia de azul $\left(-b^{*}\right)$ a amarelo $\left(+b^{*}\right)$, não demonstrou grandes variações entre os tratamentos, situando-se na faixa positiva e tendendo para o amarelo, cor característica dos bolos.

O parâmetro diferença de cor também é usado para se verificar modificações na cor causada pela adição da farinha de quinoa no bolo. Como pode ser observado na Tabela 4, houve uma tendência no aumento de $\Delta \mathrm{E}^{*}$ ao aumentar a concentração de quinoa na formulação do bolo, indicando escurecimento do mesmo.

\section{Análise sensorial}

A análise de variância mostrou não existir diferença $(p>0,05)$ entre as amostras dos bolos avaliadas quanto à aceitação sensorial, considerando os atributos aroma, cor, aparência, sabor e textura (Tabela 5). Todas as médias obtidas na aceitação sensorial localizam-se entre os pontos hedônicos 7 e 8, correspondentes aos termos "gostei ligeiramente" e "gostei muito". 
Tabela 5 - Resultados da análise sensorial de aceitação para a avaliação das amostras de bolo, considerando os atributos sabor, textura e impressão global

\begin{tabular}{|c|c|c|}
\hline \multirow{2}{*}{ Atributos } & \multicolumn{2}{|c|}{ Bolos* } \\
\hline & F10 & F20 \\
\hline Aroma & $7,2 \mathrm{a}$ & $7,1 \mathrm{a}$ \\
\hline Cor & $7,9 a$ & $7,8 \mathrm{a}$ \\
\hline Aparência & $7,7 \mathrm{a}$ & $7,7 \mathrm{a}$ \\
\hline Sabor & $7,1 \mathrm{a}$ & $7,2 \mathrm{a}$ \\
\hline Textura & $7,7 \mathrm{a}$ & $7,4 a$ \\
\hline
\end{tabular}

*Médias seguidas pela mesma letra, nas linhas, não diferem entre si pelo teste $\mathrm{F}(\mathrm{p}<0,05)$

Silva et al. (2010) também avaliaram a aceitação sensorial de bolos com diferentes níveis de substituição parcial de farinha de trigo pela farinha de quinoa $(0 \%, 10 \%, 30 \%, 50 \%)$ e verificaram que a formulação com $10 \%$ não diferiu estatisticamente do controle (100\% de farinha de trigo), sendo a mais aceita pelos provadores.

Este comportamento foi similar ao do presente estudo, quando comparado às duas concentrações de farinha de quinoa (10 e 20\%), que não apresentaram diferença significativa ( $>>0,05)$. Assim, é possível concluir que níveis de substituição de farinha de trigo pela farinha de quinoa, de até $20 \%$, não interfere na aceitação sensorial de bolos.

Assim como os bolos do presente estudo foram considerados bem aceitos pelos avaliadores, outros trabalhos realizados com o intuito de verificar a viabilidade sensorial de produtos contendo quinoa (LORENZ e COULTER, 2005; CASTRO et al., 2007; LOPES et al., 2009; KIRINUS et al., 2010; SILVA et al., 2011), obtiveram resultados semelhantes.

\section{Conclusão}

A avaliação tecnológica das farinhas mistas, compostas por farinha de trigo e quinoa integral, demonstrou características favoráveis à produção de bolos. As análises físicas dos bolos demonstraram que a adição de farinha de quinoa não alterou a estrutura interna do produto final, confirmada pela redução nos valores de densidade da massa crua e manutenção do volume do produto quando comparado ao controle. Embora as análises físicas não tenham evidenciado melhorias na aparência do produto, os bolos contendo $10 \%$ e $20 \%$ de farinha integral de quinoa obtiveram boa aceitação sensorial, considerando os atributos aroma, cor, aparência, sabor e textura. 
Conclui-se que é viável a utilização de até $20 \%$ de farinha de quinoa em substituição parcial à farinha de trigo em formulações de bolo, considerando aspectos tecnológicos e sua composição em componentes nutricionais.

\begin{abstract}
The objective of this study was to evaluate the technological and sensory feasibility of using mixed wheat and quinoa flour in the proportions of 100:00, 90:10 and 80:20 for cake production. The quinoa flour $(Q F)$ showed coarser grain size when compared to wheat flour (WF), however, there were no significant variations in the studied mixtures. It was found by flour analysis that water absorption and the time of maximum development increased $(p<0.05)$ with the increment in the content of $Q F$. There were no changes $\left(p<0^{\prime} 05\right)$ in the batter specific density, volume, symmetry and shape of cakes F1O and F20 compared to FO. The components $L^{*}$ and $b^{*}$ not vary $(p>0.05)$ between formulations, however the values of $a^{*}$ showed tendency $(p<0.05)$ to blackening with quinoa increased in the mixture. The sensory analysis showed no difference $(p>0.05)$ between the formulations with $10 \%$ and $20 \%$ of $Q F$, which had a good acceptance by consumers of cakes. The use of up to $20 \%$ of quinoa did not change the acceptance of the attributes aroma, color, appearance, flavor and texture of the cake what means to be viable using of that percentage for partial replacement of wheat flour for cake making.
\end{abstract}

Key-words: wheat, quinoa; cake; physical assessment; sensory acceptance.

\title{
Referências
}

AGÊNCIA NACIONAL DE VIGILÂNCIA SANITÁRIA DE ALIMENTOS (ANVISA). Portaria $\mathbf{n}^{\mathbf{~}} \mathbf{3 5 4}$, de $\mathbf{1 8}$ de julho de 1996. Norma técnica referente à farinha de trigo. Disponível em: <http://www.anvisa.gov.br/anvisalegis/portarias/354_96.htm>. Acesso em out. 2012.

ALMEIDA, M. L.; SANTOS, A. A. O.; SILVA, I. C. V.; LEITE, M. L. C.; SANTOS, J. P. A.; MARCELlini, P. S. Desenvolvimento de bolos de chocolate a partir da incorporação de produtos da mandioca na farinha de trigo. Scientia Plena, v. 7, n. 12, p. 1-6, 2011.

ALVES, J. S.; PEREIRA, L.; PEREIRA, J. Análises físico-químicas de pães de queijo elaborados com farinha de quinoa. XIX Congresso de Pós-Graduação da UFLA, 27 de setembro a 01 de outubro de 2010. Disponível em $<$ http://www.sbpcnet.org.br/livro/lavras/resumos/2307.pdf>. Acesso em out. 2012.

AMERICAN ASSOCIATION OF CEREAL CHEMISTS (AACC). Approved methods of the AACC. $8^{\text {th }}$ Ed. St. Paul, 1983.

AMERICAN ASSOCIATION OF CEREAL CHEMISTS (AACC). Approved methods of the AACC. $10^{\text {th }}$ Ed. St. Paul, 2000.

ASSOCIATION OF OFFICIAL AGRICULTURAL CHEMISTS (AOAC). Official Methods of Analysis of the Association of Official Agriculture Chemists. $16^{\text {th }}$ Ed. Washington, 1995. v. 2.

BORGES, J. T. S.; PIROZI, M. R.; DELlA LUCIA, S. M.; PEREIRA, P. C.; MORAES, A. R. F.; CASTRO, V. C. Utilização de farinha mista de aveia e trigo na elaboração de bolos. Boletim CEPPA, v. 24, n. 1, p. 145-162, 2006.

BORGES, J. T. S.; PIROZI, M. R.; PAULA, C. D.; RAMOS, D. L.; CHAVES, J. B. P. Caracterização físico-química e sensorial de pão de sal enriquecido com farinha integral de linhaça. Boletim CEPPA, v. 29, n. 1, p. 83-96, 2011. 
CALDERElli, V. A. S.; BENASSI, M. T.; VISENTAINER, J. V.; MATIOLI, G. Quinoa and Flaxseed: Potential Ingredients in the Production of Bread with Functional Quality. Brazilian Archives of Biology and Technology, v. 53, n. 4, p. 981-986, 2010. http://dx.doi.org/10.1590/S1516-89132010000400029

CASTRO, L.Í.A.; VILA REAL, C. M.; PIRES, I. S. C.; PIRES, C. V.; PINTO, N. A. V. D.; MIRANDA, L. S.; ROSA, B. C.; DIAS, P. A. Quinoa (Chenopodium quinoa Willd): digestibilidade in vitro, desenvolvimento e análise sensorial de preparações destinadas a pacientes celíacos. Alimentos e Nutrição, v. 18, n. 4, p. 413-419, 2007.

CAUVAIN, S. P.; YOUNG, L. S. Tecnologia da Panificação. 2a Ed. São Paulo: Editora Manole, 2009. 418p.

COELHO, L. M.; WOSIACKI, G. Avaliação sensorial de produtos panificados com adição de farinha de bagaço de maçã. Ciência e Tecnologia de Alimentos, v. 30, n. 3, p. 582-588, 2010. http://dx.doi.org/10.1590/S010120612010000300003

COSTA, M. G.; SOUZA, E. L.; STAMFORD, T. L. M.; ANDRADE, S. A. C. Qualidade tecnológica de grãos e farinhas de trigo nacionais e importados. Ciência e Tecnologia de Alimentos, v. 28, n. 1, p. 220-225, 2008. http://dx.doi.org/10.1590/S0101-20612008000100031

DA SILVA, L. M. R.; ABREU, D. A.; SOARES, D. J.; PONTES, D. F.; CONSTANT, P. B. L. Processamento de bolo com farinha de quinoa (Chenopodium quinoa Willd): estudo de aceitabilidade. Revista Brasileira de Produtos Agroindustriais, v. 12, n. 2, p. 125-132, 2010.

DANElli, D.; COSTA, G. P.; MElO, L. M.; PAGNO, C. H.; GEWEHR, M. F.; FlÔRES, S. H.; JONG, E. V. Avaliação biológica da funcionalidade de pão de fôrma com adição de quinoa (Chenopodium quinoa). Brazilian Journal of Food Technology, III SSA, p. 10-15, 2010.

FERNANDES, A. F.; PEREIRA, J.; GERMANI, R.; OIANO-NETO, J. Efeito da substituição parcial da farinha de trigo por farinha de casca de batata (Solanum Tuberosum Lineu). Ciência e Tecnologia de Alimentos, v. 28, supl., p. 56-65, 2008.

FOEGEDING, E. A.; LUCK, P. J.; DAVIS, J. P. Factors determing the physical properties of protein foams. Food Hydrocolloids, v. 20, p. 284-292, 2006. http://dx.doi.org/10.1016/j.foodhyd.2005.03.014

GEWEHR, M. F. Desenvolvimento de Pão de Forma com Adição de Quinoa. Porto Alegre, 2010. 103 f. Dissertação (Mestrado em Ciência em Tecnologia de Alimentos) - Universidade Federal do Rio Grande do Sul, Rio Grande do Sul.

GÓMEZ, M.; MORALEJA, A.; OLIETE, B.; RUIZ, E.; CABALLERO, P. A. Effect of fibre size on the quality of fibre-enriched layer cakes. LWT - Food Science and Technology, v. 43, n. 1, p. 33-38, 2010.

GORGONIO, C. M. S.; PUMAR, M.; MOTHE, C. G. Macrocospic and physiochemical characterization of a sugarless and gluten-free cake enriched with fibers made from pumpkin seed (Cucurbita maxima, L.) flour and cornstarch. Ciência e Tecnologia de Alimentos, v. 31, n. 1, p. 109-118, 2011. http://dx.doi.org/10.1590/S010120612011000100015

GUIMARÃES, R. R.; FREITAS, M. C. J.; SILVA, V. L. M. Bolos simples elaborados com farinha da entrecasca de melancia (Citrullus vulgaris, sobral): avaliação química, física e sensorial. Ciência e Tecnologia de Alimentos, v. 30, n. 2, p. 354-363, 2010. http://dx.doi.org/10.1590/S0101-20612010000200011

GUTKOSKI, L. C.; DURIGON, A.; MAZZUTTI, S.; SILVA, A. C. T.; ELIAS, M. C. Efeito do período de maturação de grãos nas propriedades físicas e reológicas de trigo. Ciência e Tecnologia de Alimentos, v. 28, n. 4, p. 888-894, 2008. http://dx.doi.org/10.1590/S0101-20612008000400019

GUTKOSKI, L. C.; DURIGON, A.; MAZZUTTI, S.; CEZARE, K.; COLLA, L. M. Influência do tipo de farinha na elaboração de bolo inglês. Brazilian Journal of Food Technology, v. 14, n. 4, p. 275-282, 2011. HTTP://DX.DOI.ORG/10.4260/BJFT2011140400033

HADNADEV, T. D.; TORBICA, A.; HADNADEV, M. Rheological properties of wheat flour substitutes/alternative crops assessed by Mixolab. Procedia - Food Science, v.1, p.328-334, 2011. 
HERA, E.; RUÍZ-PARIS, E.; OLIETE, B.; GÓMEZ, M. Studies of the quality of cakes made with wheat-lentil composite flours. LWT - Food Science and Technology, v. 49, n. 1, p. 48-54, 2012.

HUNTERLAB. User’s manual with universal software versions 3.5. Reston: HunterLab, 1998.

INDRANI, D.; RAO, G. V. Rheological characteristics of wheat flour dough as influenced by ingredients of Parotta. Journal of Food Engineering, v. 17, n. 1, p. 110-105, 2007.

KIRINUS, P.; COPETTI, C.; OLIVEIRA, V. R. Utilização de farinha de soja (Glycine max) e de quinoa (Chenopodium quinoa) no preparo de macarrão caseiro sem glúten. Alimentos e Nutrição, v. 21, n. 4, p. 555-561, 2010.

KOCER, D.; HICSASMAZ, Z.; BAYINDIRLI, A.; KATNAS, S. Bubble and pore formation of the high-ratio cake formulation with polydextrose as a sugar- and fat-replacer. Journal of Food Engineering, v. 78, p. 953-964, 2007. http://dx.doi.org/10.1016/j.jfoodeng.2005.11.034

LOPES, C. O.; DESSIMONI, G. V.; COSTA DA SILVA, M.; VIEIRA, G.; PINTO, N. A. V. D. Aproveitamento, composição nutricional e antinutricional da farinha de quinoa (Chenopodium quinoa). Alimentos e Nutrição, v. 20 , n. 4, p. 669-675, 2009.

LOPES, C. O.; ABreU, W. C.; BARCElOS, M. F. P.; PEREIRA, M. A. P.; PÍRES, C. R. F.; LIMA, J. P. Caracterização química de farinhas de quinoa (Chenopodium quinoa) submetidas a diferentes tipos de processamento. XIX Congresso de Pós-Graduação da UFLA, 27 de setembro a 01 de outubro de 2010.

LORENZ, K.; COUlTER, L. Quinoa flour in baked products. Plant Foods for Human Nutrition, v. 41, p. 213-223, 2005. http://dx.doi.org/10.1007/BF02196389

MINIM, V. P. R. Análise sensorial estudos com consumidores. 2ª Ed. Viçosa: Editora UFV, 2010. 308p.

MORAES, E. A.; DANTAS, M. I. S .; MORAIS, D. C.; SIlVA, C. O.; CASTRO, F. A. F.; MARTINO, H. S. D.; RIBEIRO, S. M. R. Sensory evaluation and nutritional value of cakes prepared with whole flaxseed flour. Ciência e Tecnologia de Alimentos, v. 30, n. 4, p. 974-979, 2010. http://dx.doi.org/10.1590/S0101-20612010000400021

NÚCLEO DE ESTUDOS E PESQUISA EM ALIMENTOS (NEPA). Tabela brasileira de composição de alimentoTACO. $4^{\text {a }}$ Ed. Campinas: NEPA-UNICAMP, 2011. 161p.

PYLER, E. J.; GORTON, L. A. Baking: science \& technology, formulation \& Production. 4 ed. Kansas: Sosland Publishing Company, 2009. V. 2. 772 p.

REPO-CARRASCO-VALENCIA, R. A. M.; SERNA, L. A. Quinoa (Chenopodium quinoa, Willd.) as a source of dietary fiber and other functional components. Ciência e Tecnologia de Alimentos, v. 31, n. 1, p. $225-230,2011$. http://dx.doi.org/10.1590/S0101-20612011000100035

SCHMIELE, M.; SILVA, L. H.; COSTA, P. F. P.; RODRIGUES, R. S.; CHANG, Y. K. Influência da adição de farinha integral de aveia, flocos de aveia e isolado proteico de soja na qualidade tecnológica de bolo inglês. Boletim CEPPA, v. 29, n. 1, p. 71-82, 2011.

SILVA, R. F.; ASCHERI, J. L. R.; PEREIRA, R. G. F. A.; MODESTA, R. C. D. Aceitabilidade de biscoitos e bolos à base de arroz com café extrusados. Ciência e Tecnologia de Alimentos, v. 29, n. 4, p. 815-819, 2009.

SILVA, F. D.; PANTE, C. F.; PRUDÊNCIO, S. H.; RIBEIRO, A. B. Establishment of a cereal bar with quinoa and its sensorial and nutritional properties. Alimentos e Nutrição, v. 22, n. 1, p. 63-69, 2011. http://dx.doi.org/10.1590/S010120612009000400018

SAS Institute. The SAS System for Windows (Statistical Analysis System), release 6.12 software. Cary, 1996.

YANG, X.; FOEGEDING, E. A. Effects of sucrose on egg white protein and whey protein isolate foams: Factors determining properties of wet and dry foams (cakes). Food Hydrocolloids, v. 24, n. 2-3, p. 227-238, 2010. http://dx.doi.org/10.1016/j.foodhyd.2009.09.011 
ZAMBRANO, F.; HIKAGE, A.; ORMENESE, R. C. C.; MONTENEGRO, F. M.; RAUEN-MIGUEL, A. M. Efeito das gomas guar e xantana em bolos como substitutos de gordura. Brazilian Journal of Food Technology, v. 8, n. 1, p. 6371, 2005.

Submetido em 21 out. 2012, Aceito para publicação em 16 jul. 2013. 